\title{
Integrative reviews in Brazilian nursing postgraduation programs: a bibliometric study
}

\author{
Uso da revisão integrativa nos cursos brasileiros de pósgraduação em enfermagem: estudo bibliométrico \\ Uso de la revisión integrativa en los cursos brasileños de postgrado en enfermería: estudio bibliométrico
}

'Escola Bahiana de Medicina e Saúde Pública. Salvador, Bahia, Brazil. "Universidade Federal da Bahia. Salvador, Bahia, Brazil. "'Centro Universitário UniRuy. Salvador, Bahia, Brazil.

How to cite this article: Nobre TCN, Rosa DOS, Heliodoro EA. Integrative reviews in Brazilian nursing postgraduation programs: a bibliometric study. Rev Bras Enferm. 2020;73(Suppl 6):e20190441. doi: http://dx.doi.org/10.1590/0034-7167-2019-0441

Corresponding author:

Tábata Cerqueira Nascimento Nobre E-mail: tabatacn@gmail.com

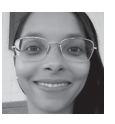

EDITOR IN CHIEF: Dulce Barbosa ASSOCIATE EDITOR: Ana Fátima Fernandes

\begin{abstract}
Objectives: to analyze the Brazilian scientific integrative review production in postgraduation nursing programs. Methods: bibliometric design, carried out in the database of dissertations and theses of the Coordination for the Improvement of Higher Education Personnel, in October 2018. Results: 35 dissertations and 1 thesis were found. Most studies were carried out at the University of São Paulo; the main methodological reference was Mendes, Silveira and Galvão (2008); for data collection, it was Ursi (2005). The most used database was CINAHL, followed by LILACS and PubMed. The period of publication varied from 5 to 30 years; and the sample, from 6 to 299 texts. Some studies did not mention keywords, data collection period, exclusion criteria or publishing period. Conclusions: despite the growing popularity of integrative review studies, they are undervalued in the stricto sensu postgraduation program in nursing and there are shortcomings in the implementation of the method, related to the absence of important elements.

Descriptors: Nursing; Review; Education, Graduate; Education, Graduate, Nursing; Bibliometrics.
\end{abstract}

\section{RESUMO}

Objetivos: analisar a produção científica brasileira, na pós-graduação em enfermagem, que utilizou o método da revisão integrativa. Métodos: bibliometria, realizada no banco de dissertações e teses da Coordenação de Aperfeiçoamento de Pessoal de Nível Superior, em outubro de 2018. Resultados: foram localizadas 35 dissertações e 1 tese. A maior produção ocorreu na Universidade de São Paulo; a principal referência metodológica foi Mendes, Silveira e Galvão (2008); e, para coleta de dados, Ursi (2005). A base mais utilizada foi CINAHL; e as plataformas, LILACS e PubMed. O recorte temporal variou de 5 a 30 anos; e a amostra, entre 6 a 299 textos. Alguns estudos não citaram descritores, período de levantamento dos dados, critérios de exclusão nem recorte temporal. Conclusões: apesar da propagação de estudos de revisão integrativa, observa-se sua subvalorização na pós-graduação stricto sensu em enfermagem e fragilidades na execução do método, com ausência de elementos importantes. Descritores: Enfermagem; Revisão; Educação Superior; Educação de Pós-Graduação em Enfermagem; Bibliometria.

\section{RESUMEN}

Objetivos: analizar la producción científica brasileña, en el postgrado en enfermería, que utilizó el método de la revisión integrativa. Métodos: bibliometría, realizada en el banco de disertaciones y tesis da la Coordinación de Perfeccionamiento de Personal de Nivel Superior, en octubre de 2018. Resultados: han sido localizadas 35 disertaciones y 1 tesis. La mayor producción ocurrió en la Universidad de São Paulo; la principal referencia metodológica ha sido Mendes, Silveira y Galvão (2008); y, para recogida de datos, Ursi (2005). La base más utilizada ha sido CINAHL; y las plataformas, LILACS y PubMed. El recorte temporal ha variado de 5 a 30 años; y la muestra, entre 6 a 299 textos. Algunos estudios no citaron descriptores, período de levantamiento de los datos, criterios de exclusión ni recorte temporal. Conclusiones: a pesar de la propagación de estudios de revisión integrativa, se observa su subvalorización en el postgrado stricto sensu en enfermería y fragilidades en la ejecución del método, con ausencia de elementos importantes.

Descriptores: Enfermería; Revisión; Educación Superior; Educación de Postgrado en Enfermería. 


\section{INTRODUCTION}

Integrative reviews present, in an orderly manner, research results on a specific topic, with the aim of facilitating the use of scientific evidence in clinical practice. They started to be reported as a research method in 1980(1), but until 1998 there were no

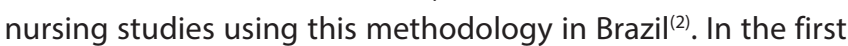
decade of the 2000s, pioneering studies began to emerge, with great repercussions in the nursing area ${ }^{(3)}$. It was also during this period that national productions emerged with information on the steps for conducting research using this methodology, offering theoretical support to researchers ${ }^{(1)}$.

Despite this growth, there are still difficulties in carrying out this type of study, mainly in differentiating it from systematic reviews and in the operationalization of its basic steps. Both have a delimited theme, start from a research question, and have a defined protocol to replicate the study and reduce bias. The particularity of the integrative review is that it includes studies with different methodological approaches, whether qualitative or quantitative, covering a broader range on the studied subject ${ }^{(4)}$.

This type of research reached lato sensu postgraduation courses, and even stricto sensu ones, since the number of courses and students requiring tutelage increased, the time to develop the study diminished, scientific production increased, and access was made easier through the internet. Integrative reviews are also to be predecessors to the production of primary studies, since they support the assessment of the current knowledge on the subject and help to clearly define the objects of studies. In many studies, at this category of formation, there is a chapter, session or even an article dedicated to the review phase $\mathrm{e}^{(5)}$. Therefore, this research raises the following question: What is the current situation of scientific production in Brazilian postgraduation courses in nursing that use integrative reviews as a method?

\section{OBJECTIVES}

To analyze the Brazilian scientific integrative review production in postgraduation nursing programs.

\section{METHODS}

\section{Ethical aspects}

The research was not submitted to the Human Research Ethics Committee since it was carried out with secondary and public domain data. However, the copyright preservation of the studies was considered.

\section{Study design, period and location}

This is a bibliometric study that, through mathematical techniques, evaluates the scientific production of several areas of knowledge. It considers databases, dissertations and theses, as they are able to provide information for building an overview of the theme ${ }^{(6)}$.

The data was collected at the Theses and Dissertations database of the Coordination for the Improvement of Higher Education Personnel (CAPES). These types of work were chosen because they comprise a high level of scientific production in the country, presenting methodological rigor and being validated in evaluation boards. Data were collected in October 2018.

In the search strategy, the controlled keyword "Review" was used, as established by the database Health Sciences Descriptors (DeCS). In order to refine the search, the keyword "Integrative" was added, despite not being a standardized term. As a resource, the expression in quotation marks and the database own filters were used, selecting "Nursing" as the area of knowledge.

\section{Population or sample; inclusion and exclusion criteria}

The inclusion criteria were theses or dissertations, available in full, which used the integrative review as their only research methodology. Completed and approved studies from 2013 were included, as did those that were available in full on the Portal up to 2017. Researches with other types of review or who did the integrative review to support an original research were excluded.

\section{Study protocol}

In the first stage, through the use of the expression "Integrative Review", 862 studies were found. After filtering by the field of knowledge (nursing) and year, 336 remained. The abstracts were read to identify if the main objective of the study was to carry out an integrative review and if they met the other inclusion and exclusion criteria. After selecting the studies, data was extracted and organized in a Microsoft Excel ${ }^{\circ}$ spreadsheet, containing the variables of interest.

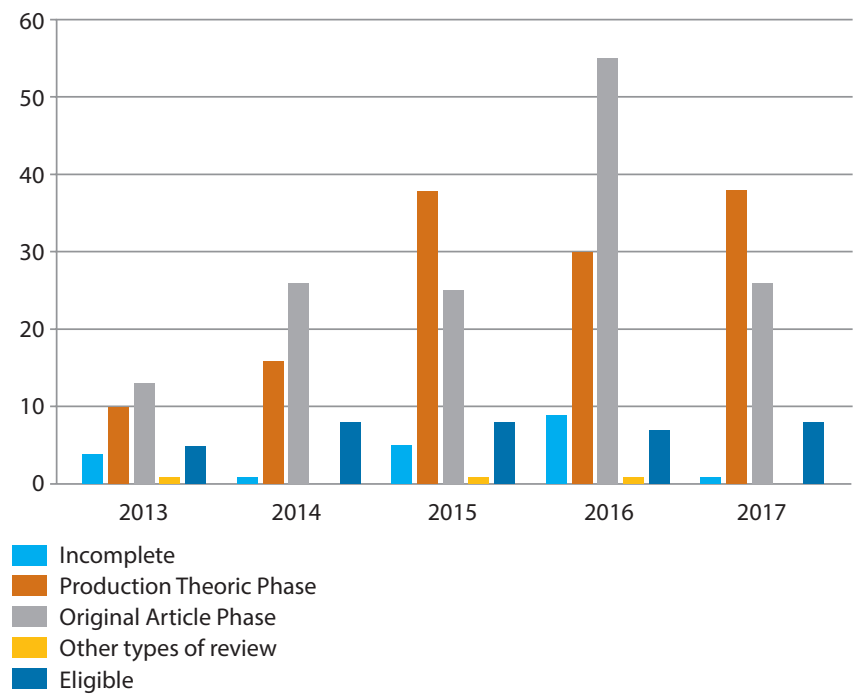

Source: Dissertations and thesis defended between 2013 and 2017 that used the integrative review methodology.

Figure 1 - Sample Selection

\section{Statistics and analysis of results}

The information contained in the spreadsheet was analyzed individually, composing the study variables. Data was analyzed descriptively and presented in the form of tables and graphs, according to the specificity of each information. 


\section{RESULTS}

This review included 35 dissertations, 7 of which were from a professional master's program, and 1 thesis. The studied contents were classified according to the keyword. A diversity of areas of interest was observed: healthcare to people deprived of liberty ${ }^{(7-8)}$; home care ${ }^{(9)}$; infections $^{(10-11)}$; dialysis ${ }^{(12)}$; hypertension ${ }^{(13-14)}$; tuberculosis ${ }^{(15-16)}$; nursing education ${ }^{(17-20)}$; care management $^{(21)}$; cancer $^{(2223)}$; child health ${ }^{(24)}$; domestic violence ${ }^{(25)}$; falls in elderly and psychiatric patients ${ }^{(26-28)}$; nursing work ${ }^{(29-30)}$; patient classification ${ }^{(31)}$; clinical reasoning ${ }^{(32)}$; marijuana legalization ${ }^{(33)}$; welders $^{(34)}$; ulcers $^{(35)}$; perioperative nursing ${ }^{(36)}$; sexuality $^{(37)}$; mental health ${ }^{(38)}$; abuse of psychoactive substances ${ }^{(39)}$; medication errors $^{(40)}$; hospital accreditation ${ }^{(4))}$; family health strategy ${ }^{(42)}$.

Chart 1 - Information of the selected dissertations and thesis, Salvador, Bahia, Brazil, 2019

\begin{tabular}{|c|c|c|c|c|}
\hline Title & Author & Year & $\begin{array}{l}\text { Type of } \\
\text { study }\end{array}$ & University \\
\hline $\begin{array}{l}\text { Nursing interventions } \\
\text { for treatment of pressure } \\
\text { ulcer in bedridden } \\
\text { patients: integrative } \\
\text { literature review }{ }^{(35)}\end{array}$ & $\begin{array}{l}\text { Ana } \\
\text { Débora } \\
\text { Alcântara } \\
\text { Coelho }\end{array}$ & 2013 & Dissertation & $\begin{array}{l}\text { Universidade } \\
\text { Federal do } \\
\text { Ceará }\end{array}$ \\
\hline $\begin{array}{l}\text { Nurses job satisfaction } \\
\text { and dissatisfaction: } \\
\text { literature integrative } \\
\text { review }^{(29)}\end{array}$ & $\begin{array}{l}\text { Isabela } \\
\text { Saura } \\
\text { Sartoreto }\end{array}$ & 2013 & Dissertation & $\begin{array}{l}\text { Universidade } \\
\text { de São Paulo }\end{array}$ \\
\hline $\begin{array}{l}\text { Teaching methods in the } \\
\text { prevention of catheter- } \\
\text { related bloodstream } \\
\text { infection: an integrative } \\
\text { review of literature }{ }^{(17)}\end{array}$ & $\begin{array}{l}\text { Christian } \\
\text { Emmanuel } \\
\text { da Silva } \\
\text { Pelaes }\end{array}$ & 2013 & Dissertation & $\begin{array}{l}\text { Universidade } \\
\text { de São Paulo }\end{array}$ \\
\hline $\begin{array}{l}\text { Strategies for reducing } \\
\text { stress and its impact } \\
\text { on the immune system } \\
\text { of women with breast } \\
\text { cancer: evidence for } \\
\text { clinical practice }^{(23)}\end{array}$ & $\begin{array}{l}\text { Camila } \\
\text { Aparecida } \\
\text { Abrahão }\end{array}$ & 2013 & Dissertation & $\begin{array}{l}\text { Universidade } \\
\text { de São Paulo/ } \\
\text { Ribeirão } \\
\text { Preto }\end{array}$ \\
\hline $\begin{array}{l}\text { Scientific on falls in the } \\
\text { elderly: international } \\
\text { components for } \\
\text { the classification of } \\
\text { functioning, disability, } \\
\text { and health } \\
\text { (26) }\end{array}$ & $\begin{array}{l}\text { Julia de } \\
\text { Moura } \\
\text { Quintana }\end{array}$ & 2013 & Dissertation & $\begin{array}{c}\text { Universidade } \\
\text { Federal do } \\
\text { Rio Grande }\end{array}$ \\
\hline $\begin{array}{l}\text { Tuberculosis and } \\
\text { diabetes mellitus: } \\
\text { approaches in spatio- } \\
\text { temporal trajectory }{ }^{(15)}\end{array}$ & $\begin{array}{l}\text { Anna } \\
\text { Carolina } \\
\text { Lima de } \\
\text { Alencar }\end{array}$ & 2014 & Dissertation & $\begin{array}{l}\text { Universidade } \\
\text { de São Paulo }\end{array}$ \\
\hline $\begin{array}{l}\text { Knowledge produced } \\
\text { by Brazilian nurses on } \\
\text { arterial hypertension: } \\
\text { an integrative review } \\
\text { study }{ }^{(13)}\end{array}$ & $\begin{array}{l}\text { Victor Cauê } \\
\text { Lopes }\end{array}$ & 2014 & Dissertation & $\begin{array}{c}\text { Universidade } \\
\text { Guarulhos }\end{array}$ \\
\hline $\begin{array}{l}\text { State of the art of the } \\
\text { event falls in elders: an } \\
\text { integrative literature } \\
\text { review } \\
\text { (27) }\end{array}$ & $\begin{array}{l}\text { Newton } \\
\text { Ferreira } \\
\text { de Paula } \\
\text { Junior }\end{array}$ & 2014 & Dissertation & $\begin{array}{l}\text { Universidade } \\
\text { Federal } \\
\text { de Santa } \\
\text { Catarina }\end{array}$ \\
\hline $\begin{array}{l}\text { Care management by } \\
\text { Brazilian nurses: an } \\
\text { integrative literature } \\
\text { review }^{(21)}\end{array}$ & $\begin{array}{c}\text { Wedja } \\
\text { Cristina do } \\
\text { O'Oliveira } \\
\text { Correa }\end{array}$ & 2014 & $\begin{array}{c}\text { Dissertation } \\
\text { - Professional } \\
\text { Master's } \\
\text { degree }\end{array}$ & $\begin{array}{c}\text { Universidade } \\
\text { Estadual } \\
\text { de Feira de } \\
\text { Santana }\end{array}$ \\
\hline
\end{tabular}

To be continued
Chart 1

\begin{tabular}{|c|c|c|c|c|}
\hline Title & Author & Year & $\begin{array}{l}\text { Type of } \\
\text { study }\end{array}$ & University \\
\hline $\begin{array}{l}\text { Nursing care for the } \\
\text { liver transplant recipient } \\
\text { in the postoperative } \\
\text { period: an integrative } \\
\text { review }^{(36)}\end{array}$ & $\begin{array}{l}\text { Fabiana } \\
\text { Murad } \\
\text { Rossin } \\
\text { Molina }\end{array}$ & 2014 & Dissertation & $\begin{array}{l}\text { Universidade } \\
\text { de São Paulo/ } \\
\text { Ribeirão } \\
\text { Preto }\end{array}$ \\
\hline $\begin{array}{l}\text { Characterization of } \\
\text { health care provided } \\
\text { to persons deprived of } \\
\text { liberty who live with } \\
\text { HIV/AIDS: an integrative } \\
\text { review }^{(7)}\end{array}$ & $\begin{array}{l}\text { Erika } \\
\text { Aparecida } \\
\text { Catoia }\end{array}$ & 2014 & Dissertation & $\begin{array}{l}\text { Universidade } \\
\text { de São Paulo/ } \\
\text { Ribeirão } \\
\text { Preto, }\end{array}$ \\
\hline $\begin{array}{l}\text { Use of patients' } \\
\text { classification } \\
\text { instruments: analysis of } \\
\text { the Brazilian production } \\
\text { of knowledge }^{(31)}\end{array}$ & $\begin{array}{l}\text { Sonia } \\
\text { Portella de } \\
\text { Abreu }\end{array}$ & 2014 & Dissertation & $\begin{array}{l}\text { Faculdade de } \\
\text { Medicina de } \\
\text { São José do } \\
\text { Rio Preto }\end{array}$ \\
\hline $\begin{array}{l}\text { Arterial hypertension } \\
\text { in black populations: } \\
\text { an analysis of articles } \\
\text { published by nurses in } \\
\text { Brazil and in the United } \\
\text { States }^{(14)}\end{array}$ & $\begin{array}{l}\text { Alessandra } \\
\text { Ferreira } \\
\text { Mendes } \\
\text { Jiticovski }\end{array}$ & 2014 & Dissertation & $\begin{array}{c}\text { Universidade } \\
\text { Guarulhos }\end{array}$ \\
\hline $\begin{array}{l}\text { Magnet recognition } \\
\text { program: an integrative } \\
\text { literature review } \\
\text { (41) }\end{array}$ & $\begin{array}{l}\text { Thais } \\
\text { Cristina de } \\
\text { Hollanda } \\
\text { Parisi }\end{array}$ & 2015 & Dissertation & $\begin{array}{l}\text { Universidade } \\
\text { de São Paulo }\end{array}$ \\
\hline $\begin{array}{l}\text { Singular therapeutic } \\
\text { project: integrative } \\
\text { literature review }{ }^{(38)}\end{array}$ & $\begin{array}{l}\text { Livia } \\
\text { Gouveia da } \\
\text { Silva }\end{array}$ & 2015 & Dissertation & $\begin{array}{l}\text { Universidade } \\
\text { de São Paulo }\end{array}$ \\
\hline $\begin{array}{l}\text { Strategies for the } \\
\text { control of tuberculosis } \\
\text { in the prison system: } \\
\text { integrative literature } \\
\text { review }^{(16)}\end{array}$ & $\begin{array}{l}\text { Monica } \\
\text { Cristina } \\
\text { Ribeiro } \\
\text { Alexandre } \\
\text { Dauria de } \\
\text { Lima }\end{array}$ & 2015 & Dissertation & $\begin{array}{l}\text { Universidade } \\
\text { de São Paulo } \\
\text { /Ribeirão } \\
\text { Preto }\end{array}$ \\
\hline $\begin{array}{l}\text { The early stress as } \\
\text { a determinant of } \\
\text { the abusive use } \\
\text { of psychoactive } \\
\text { substances: an } \\
\text { integrative review(39) }\end{array}$ & $\begin{array}{l}\text { Larissa } \\
\text { Bessani } \\
\text { Hidalgo } \\
\text { Gimenez }\end{array}$ & 2015 & Dissertation & $\begin{array}{l}\text { Universidade } \\
\text { de São Paulo } \\
\text { /Ribeirão } \\
\text { Preto }\end{array}$ \\
\hline $\begin{array}{l}\text { Technology in health } \\
\text { and safety drug } \\
\text { administration in } \\
\text { hospitalized patients: an } \\
\text { integrative review }{ }^{(40)}\end{array}$ & $\begin{array}{l}\text { Paula de } \\
\text { Siqueira } \\
\text { Furquim }\end{array}$ & 2015 & $\begin{array}{c}\text { Dissertation } \\
\text { - Professional } \\
\text { Master's } \\
\text { degree }\end{array}$ & $\begin{array}{l}\text { Universidade } \\
\text { de São Paulo/ } \\
\text { Ribeirão } \\
\text { Preto }\end{array}$ \\
\hline $\begin{array}{l}\text { Educational digital } \\
\text { technology and the } \\
\text { clinical case study } \\
\text { for nursing students' } \\
\text { learning }\end{array}$ & $\begin{array}{c}\text { Cristina } \\
\text { Yuri Nakata } \\
\text { Hara }\end{array}$ & 2015 & Dissertation & $\begin{array}{l}\text { Universidade } \\
\text { de São Paulo/ } \\
\text { Ribeirão } \\
\text { Preto }\end{array}$ \\
\hline $\begin{array}{l}\text { Evidences associated to } \\
\text { the nursing intervention } \\
\text { "infection control" from } \\
\text { the classification of } \\
\text { nursing interventions: } \\
\text { guidance and teaching } \\
\text { on washing the hands }{ }^{(10)}\end{array}$ & $\begin{array}{l}\text { Lilian } \\
\text { Salgado } \\
\text { Cunha } \\
\text { Brito }\end{array}$ & 2015 & $\begin{array}{c}\text { Dissertation } \\
\text { - Professional } \\
\text { Master's } \\
\text { degree }\end{array}$ & $\begin{array}{l}\text { Universidade } \\
\text { Estadual } \\
\text { Paulista Júlio } \\
\text { de Mesquita } \\
\text { Filho/ } \\
\text { Botucatu }\end{array}$ \\
\hline $\begin{array}{l}\text { Infection postpartum } \\
\text { period: implications for } \\
\text { nursing }^{(11)}\end{array}$ & $\begin{array}{l}\text { Leila } \\
\text { Jussara } \\
\text { Berlet }\end{array}$ & 2015 & Dissertation & $\begin{array}{l}\text { Universidade } \\
\text { do Estado do } \\
\text { Rio de Janeiro }\end{array}$ \\
\hline $\begin{array}{l}\text { Violence against women } \\
\text { before incarceration: } \\
\text { integrative review of } \\
\text { literature }^{(8)}\end{array}$ & $\begin{array}{l}\text { Fabiola } \\
\text { Meirelles } \\
\text { Israel } \\
\text { Pessoa }\end{array}$ & 2016 & Dissertation & $\begin{array}{l}\text { Universidade } \\
\text { de São Paulo/ } \\
\text { Ribeirão } \\
\text { Preto }\end{array}$ \\
\hline
\end{tabular}


Chart 1 (concluded)

\begin{tabular}{|c|c|c|c|c|}
\hline Title & Author & Year & $\begin{array}{l}\text { Type of } \\
\text { study }\end{array}$ & University \\
\hline $\begin{array}{l}\text { Spirituality for women } \\
\text { with breast cancer: an } \\
\text { integrative review } 222)\end{array}$ & $\begin{array}{l}\text { Felipe de } \\
\text { Souza } \\
\text { Areco }\end{array}$ & 2016 & Dissertation & $\begin{array}{c}\text { Universidade } \\
\text { de São Paulo/ } \\
\text { Ribeirão } \\
\text { Preto }\end{array}$ \\
\hline $\begin{array}{l}\text { The clinical reasoning } \\
\text { and its application to the } \\
\text { process of diagnosing } \\
\text { nursing: integrative } \\
\text { review of literature }{ }^{(32)}\end{array}$ & $\begin{array}{l}\text { Danielle } \\
\text { Dias } \\
\text { Correia da } \\
\text { Silva }\end{array}$ & 2016 & Dissertation & $\begin{array}{l}\text { Universidade } \\
\text { do Estado do } \\
\text { Rio de Janeiro }\end{array}$ \\
\hline $\begin{array}{l}\text { The legalization of } \\
\text { marijuana: a review(33) }\end{array}$ & $\begin{array}{l}\text { Anderson } \\
\text { Fabio } \\
\text { Moura } \\
\text { Weiber }\end{array}$ & 2016 & Dissertation & $\begin{array}{c}\text { Universidade } \\
\text { Estadual do } \\
\text { Sudoeste da } \\
\text { Bahia }\end{array}$ \\
\hline $\begin{array}{l}\text { Recommendations/ } \\
\text { actions of nursing } \\
\text { care to adult patients } \\
\text { submitted to } \\
\text { replacement renal } \\
\text { therapy in an intensive } \\
\text { care unit }^{(12)}\end{array}$ & $\begin{array}{l}\text { Tatiana } \\
\text { Rassele }\end{array}$ & 2016 & $\begin{array}{c}\text { Dissertation } \\
\text { - Professional } \\
\text { Master's } \\
\text { degree }\end{array}$ & $\begin{array}{l}\text { Universidade } \\
\text { Federal } \\
\text { de Santa } \\
\text { Catarina }\end{array}$ \\
\hline $\begin{array}{l}\text { The nurse working } \\
\text { process in different } \\
\text { countries }^{(30)}\end{array}$ & $\begin{array}{l}\text { Juliana } \\
\text { Alves Leite } \\
\text { Leal }\end{array}$ & 2016 & Thesis & $\begin{array}{l}\text { Universidade } \\
\text { Federal da } \\
\text { Bahia }\end{array}$ \\
\hline $\begin{array}{l}\text { Welders'health care in } \\
\text { shipyards Brazilians: an } \\
\text { integrative review }{ }^{(34)}\end{array}$ & $\begin{array}{l}\text { Marco } \\
\text { Antonio } \\
\text { Pinto } \\
\text { Balthazar }\end{array}$ & 2016 & Dissertation & $\begin{array}{l}\text { Universidade } \\
\text { Federal } \\
\text { Fluminense }\end{array}$ \\
\hline $\begin{array}{l}\text { Falls in psychiatric } \\
\text { patients: an integrative } \\
\text { review }^{(28)}\end{array}$ & $\begin{array}{l}\text { Talita } \\
\text { Portela } \\
\text { Cassola }\end{array}$ & 2017 & Dissertation & $\begin{array}{c}\text { Universidade } \\
\text { Federal do } \\
\text { Rio Grande } \\
\text { do Sul } \\
\end{array}$ \\
\hline $\begin{array}{l}\text { Sexuality of liver } \\
\text { transplantation } \\
\text { candidates and } \\
\text { recipients: an integrative } \\
\text { review of the literature }{ }^{(37)}\end{array}$ & $\begin{array}{l}\text { Jennifer } \\
\text { Tatisa } \\
\text { Jubileu } \\
\text { Magro }\end{array}$ & 2017 & Dissertation & $\begin{array}{l}\text { Universidade } \\
\text { de São Paulo } \\
\text { /Ribeirão } \\
\text { Preto }\end{array}$ \\
\hline $\begin{array}{l}\text { Educational practices } \\
\text { for nurses in emergency } \\
\text { services: an integrative } \\
\text { review }^{(2)}\end{array}$ & $\begin{array}{l}\text { Angélica } \\
\text { Salete da } \\
\text { Rosa }\end{array}$ & 2017 & \begin{tabular}{|c|} 
Dissertation \\
- Professional \\
Master's \\
degree \\
\end{tabular} & $\begin{array}{l}\text { Universidade } \\
\text { do Vale do } \\
\text { Rio dos Sinos }\end{array}$ \\
\hline $\begin{array}{l}\text { Teaching strategies } \\
\text { without development } \\
\text { of critical thinking in } \\
\text { nursing: integrated } \\
\text { review }{ }^{(19)}\end{array}$ & $\begin{array}{l}\text { Marina } \\
\text { Liberale }\end{array}$ & 2017 & Dissertation & $\begin{array}{l}\text { Universidade } \\
\text { de São Paulo } \\
\text { /Ribeirão } \\
\text { Preto }\end{array}$ \\
\hline $\begin{array}{l}\text { Responsible discharge: } \\
\text { in search of continuity } \\
\text { of care for patients in } \\
\text { palliative care at home, } \\
\text { an integrative review }\end{array}$ & $\begin{array}{l}\text { Denise } \\
\text { Sarreta } \\
\text { Ignacio }\end{array}$ & 2017 & $\begin{array}{c}\text { Dissertation } \\
\text { - Professional } \\
\text { Master's } \\
\text { degree }\end{array}$ & $\begin{array}{l}\text { Universidade } \\
\text { de São Paulo } \\
\text { /Ribeirão } \\
\text { Preto }\end{array}$ \\
\hline $\begin{array}{l}\text { Denver Il: discrepancies } \\
\text { in the assessments of } \\
\text { the development of } \\
\text { Brazilian children }^{(24)}\end{array}$ & $\begin{array}{l}\text { Yasmin } \\
\text { Souza } \\
\text { Bastos }\end{array}$ & 2017 & Dissertation & $\begin{array}{c}\text { Universidade } \\
\text { Universus } \\
\text { Veritas } \\
\text { Guarulhos }\end{array}$ \\
\hline $\begin{array}{l}\text { Matrixial support } \\
\text { in mental health: a } \\
\text { possibility of permanent } \\
\text { education? } ?^{(42)}\end{array}$ & $\begin{array}{l}\text { Rodrigo } \\
\text { Oliveira de } \\
\text { Carvalho } \\
\text { da Silva }\end{array}$ & 2017 & $\begin{array}{c}\text { Dissertation } \\
\text { - Professional } \\
\text { Master's } \\
\text { degree }\end{array}$ & $\begin{array}{l}\text { Universidade } \\
\text { Federal } \\
\text { Fluminense }\end{array}$ \\
\hline $\begin{array}{l}\text { Strategies of the } \\
\text { health sector in the } \\
\text { confrontation of } \\
\text { domestic violence } \\
\text { against children }\end{array}$ & $\begin{array}{l}\text { Rita de } \\
\text { Cassia } \\
\text { Batista de } \\
\text { Oliveira } \\
\text { Peixoto }\end{array}$ & 2017 & Dissertation & $\begin{array}{l}\text { Universidade } \\
\text { Universus } \\
\text { Veritas } \\
\text { Guarulhos }\end{array}$ \\
\hline
\end{tabular}

Source: Dissertations and thesis defended between 2013 and 2017 that used the integrative review methodology.
The studies originated from several different institutions, with the Universidade of São Paulo, including the campus in the city of Ribeirão Preto, representing $47.2 \%$ of them.

Table 1 - Distribution of dissertations and theses by educational institutions and year, Salvador, Bahia, Brazil, 2019

\begin{tabular}{|c|c|c|c|c|c|c|}
\hline Educational Institution & 2017 & 2016 & 2015 & 2014 & 2013 & TOTAL \\
\hline $\begin{array}{l}\text { Universidade de São Paulo/ } \\
\text { Ribeirão Preto }\end{array}$ & 3 & 2 & 6 & 3 & 3 & 17 \\
\hline $\begin{array}{l}\text { Universidade Universus Veritas } \\
\text { de Guarulhos }\end{array}$ & 2 & & & 2 & & 4 \\
\hline $\begin{array}{l}\text { Universidade Federal de Santa } \\
\text { Catarina }\end{array}$ & & 1 & & 1 & & 2 \\
\hline $\begin{array}{l}\text { Universidade Federal } \\
\text { Fluminense }\end{array}$ & 1 & 1 & & & & 2 \\
\hline $\begin{array}{l}\text { Universidade do Estado do Rio } \\
\text { de Janeiro }\end{array}$ & & 1 & 1 & & & 2 \\
\hline $\begin{array}{l}\text { Universidade Estadual do } \\
\text { Sudoeste da Bahia }\end{array}$ & & 1 & & & & 1 \\
\hline Universidade Federal da Bahia & & 1 & & & & 1 \\
\hline $\begin{array}{l}\text { Universidade Estadual } \\
\text { Paulista }\end{array}$ & & & 1 & & & 1 \\
\hline $\begin{array}{l}\text { Universidade Estadual de Feira } \\
\text { de Santana }\end{array}$ & & & & 1 & & 1 \\
\hline $\begin{array}{l}\text { Faculdade de Medicina de São } \\
\text { José do Rio Preto }\end{array}$ & & & & 1 & & 1 \\
\hline Universidade Federal do Ceará & & & & & 1 & 1 \\
\hline $\begin{array}{l}\text { Universidade Federal do Rio } \\
\text { Grande do Norte }\end{array}$ & & & & & 1 & 1 \\
\hline $\begin{array}{l}\text { Universidade Federal do Rio } \\
\text { Grande do Sul }\end{array}$ & 1 & & & & & 1 \\
\hline $\begin{array}{l}\text { Universidade do Vale do Rio } \\
\text { dos Sinos }\end{array}$ & 1 & & & & & 1 \\
\hline
\end{tabular}

Source: Dissertations and thesis defended between 2013 and 2017 that used the integrative review methodology.

The studied contents were classified according to the keyword. A diversity of areas of interest was observed: healthcare to people deprived of liberty ${ }^{(7-8)}$; home care $^{(9)}$; infections ${ }^{(10-11)}$; dialysis $^{(12)}$; hypertension ${ }^{(13-14)}$; tuberculosis ${ }^{(15-16)}$; nursing education ${ }^{(17-20)}$; care management ${ }^{(21)}$; cancer $^{(2223)}$; child health ${ }^{(24)}$; domestic violence ${ }^{(25) \text {; }}$ falls in elderly and psychiatric patients ${ }^{(26-28)}$; nursing work ${ }^{(29-30)}$; patient classification ${ }^{(31)}$; clinical reasoning ${ }^{(32)}$; marijuana legalization $^{(33)}$; welders $^{(34)}$; ulcers $^{(35)}$; perioperative nursing ${ }^{(36)}$; sexuality $^{(37) ;}$ mental health ${ }^{(38)}$; abuse of psychoactive substances ${ }^{(39)}$; medication errors ${ }^{(40)}$; hospital accreditation ${ }^{(41)}$; family health strategy ${ }^{(42)}$.

The theoretical framework used as methodological support in the studies has similar features, but Mendes, Silveira and Galvão (2008) were cited in 12 studies $^{(7-8,12,17,19,22,25,32,36-39)}$. Others authors used were: Whittemore and Knafl (2005), in 6 works $^{(10,13,23,28,31,35)}$; Ganong (1987), in five (8,23,27,39,41); Cooper (1989), in two $^{(18,26)}$. The following authors were used in one study each: Botelho, Cunha and Macedo (2011) ${ }^{(42)}$, Bellucci Júnior and Matsuda (2011) ${ }^{(33)}$, Broome (1993) $)^{(8)}$, Carneiro, Brito and Santos (2011) (24), Polit and Beck (2006) ${ }^{(15)}$, Beyea and Nicoll (1998)(39) and Jackson (1980)(29). Some works cited more than one author ${ }^{(8,23,39)}$, and nine did not specify the theoretical framework used ${ }^{(9,11,15-16,20-21,30,34,40)}$. 
Table 2 - Distribution of databases by year, Salvador, Bahia, Brazil, 2019

\begin{tabular}{|c|c|c|c|c|c|c|}
\hline Database used & 2017 & 2016 & 2015 & 2014 & 2013 & TOTAL \\
\hline LILACS & 5 & 7 & 6 & 8 & 5 & 31 \\
\hline PUBMED & 4 & 5 & 6 & 5 & 3 & 23 \\
\hline CINAHL & 4 & 3 & 6 & 6 & 4 & 23 \\
\hline MEDLINE & 3 & 4 & 2 & 4 & 2 & 15 \\
\hline SCIELO & 2 & 3 & & 4 & 2 & 11 \\
\hline WEB OF SCIENCE & 1 & 2 & 3 & 2 & 1 & 9 \\
\hline BDENF & 1 & 1 & 1 & 2 & & 5 \\
\hline COCHRANE & & & 1 & 1 & 3 & 5 \\
\hline SCOPUS & & 2 & 2 & 1 & 1 & 6 \\
\hline PSYCINFO & & 2 & 1 & & & 3 \\
\hline BANCOS DE TESES CAPES & & 3 & 1 & & & 4 \\
\hline BVS & 3 & & 1 & & & 4 \\
\hline EMBASE & 1 & & & & 2 & 3 \\
\hline ERIC PROQUEST & 1 & & & & 1 & 2 \\
\hline SCIENCE DIRECT & & 1 & 1 & & & 2 \\
\hline SIRIUS/UERJ & & 1 & & & & 1 \\
\hline BIREME & & 1 & & & & 1 \\
\hline BANCO DE TESES DA USP & & & 1 & & & 1 \\
\hline GOOGLE ACADÊMICO & & & & 1 & & 1 \\
\hline DEDALUS/USP & & & & 1 & & 1 \\
\hline REVISTA ESTIMA & & & & & 1 & 1 \\
\hline PERIENF & & & & & 1 & 1 \\
\hline CUIDEN & & & & & 1 & 1 \\
\hline IBESC & & & & & 1 & 1 \\
\hline $\begin{array}{l}\text { BANCO DE TESES } \\
\text { INTERNACIONAIS }\end{array}$ & & 1 & & & & 1 \\
\hline EBSCO & & & & 1 & & 1 \\
\hline $\mathrm{NCBI}$ & & & & 1 & & 1 \\
\hline
\end{tabular}

Source: Dissertations and thesis defended between 2013 and 2017 that used the integrative review methodology.

Note: LILACS - Latin American and Caribbean Literature in Health Sciences; CINAHL - Cumulative Index to Nursing and Allied Health Literature; MEDLINE - Medical Literature Analysis and Retrieval System Online; SCIELO-Scientific Electronic Library Online; BDENF-Nursing Database; BVS - Virtual Health Library; ERIC PROQUEST - Education Resources Information Center; SIRIUS/UERJ - Sirius Network of Libraries at the State University of Rio de Janeiro; BIREME-Regional Library of Medicine; USP - University of São Paulo; DEDALUS/USP - bibliographic database of the University of São Paulo; IBESC - Spanish Bibliographic Index in Health Sciences; EBSCO - Business Source Complete; NCBI - National Center for Biotechnological Information.

27 different databases and sites were found in the studies, with a predominance of three: LILACS, mentioned by $86.1 \%$; and PubMed and CINAHL, used by $63.8 \%$ of the studies.

22 studies mentioned the time period when their data collection took place ${ }^{(7,9-12,15-17,21,23,25,27-31,33-34,38-41)}$, and 20 authors included a chapter describing theoretical framework ${ }^{(7,11-12,15,17-18,20-23,26-28,30,32,34-}$ 35,38-40). Among the studies, $1^{(42)}$ made no reference to the keywords used, and $11^{(8,10-12,15,17,22,26,33-35)}$ chose to use only keywords without association with non-standardized terms. All studies surveyed presented defined inclusion criteria for the selection of articles. The exclusion criteria were not mentioned in four of the studies $(20,24,26,28)$.

Regarding the time frame of the study, the shortest was 5 years, in 12 studies $^{(8,11,16,19-20,22,26,32-34,40,42)}$; and the largest, was 30 years, used in one study ${ }^{(13)}$. The other works used the following time frames: 11 studies used the period of 10 years $^{(9-10,21,27,29,31,35-37,39,41)}$; one used 11 years ${ }^{(12)}$; one used 12 years ${ }^{(15)}$; two considered 20 years ${ }^{(23-24)}$; and six studies did not used a limited time frame ${ }^{(14,17-}$ ${ }^{18,25,28,38)}$. Two studies did not mention a time frame ${ }^{(7,30)}$.

Only six studies did not use tables with a list and synthesis of the articles included in the review ${ }^{(18-19,25,27,32-33)}$. The use of a graphic representation (flowchart) to describe the sample selection steps was absent in 10 studies $^{(7,9-10,12,14,29,33,35-36,41)}$, and the detailed presentation of a table was present in 18 studies $^{(7-9,11,16,18-20,22-23,29,32,36-41)}$.
The number of articles used in the reviews varied, with six being the smallest sample ${ }^{(34)}$, while the largest was $299^{(13)}$.

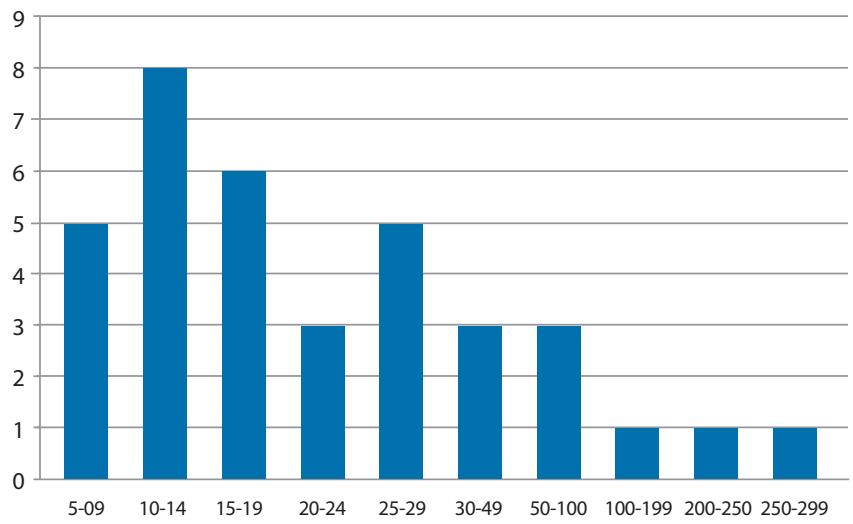

Source: Dissertations and thesis defended between 2013 and 2017 that used the integrative review methodology.

Figure 2 - Number of articles used

Only nine (25\%) studies $^{(8,15-16,20,22,27-28,30,39)}$ cited ethical aspects. Data extraction followed several strategies, with emphasis on the creation of original instruments, in 16 of the studies $^{(13-14,16,20,24-28,30,32-35,38,42)}$; seven used the complete URSI instrument ${ }^{(21,22-23,36-37,39-40)}$, and 8 adapted an instrument to elaborate their own ${ }^{(7-9,12,17-19,29)}$. The Matrix Research Appraisal Checklist method, an instrument adapted from Tronchin (2011), Romanzini (2013), Otrenti (2011) and an instrument adapted from Souza, Silva and Carvalho (2010) were each used in one study ${ }^{(11,18,29,31,41)}$. Two studies did not specify the instruments used ${ }^{(10,15)}$.

It was also found that many results were presented through categories, and only eight were not $^{(7-9,12,17,22,24,42)}$. The number of pages ranged from $47^{(25)}$ to $222^{(27)}$, with most varying from 70 to 89 pages.

\section{DISCUSSION}

In the results, among the 36 studies, there was only one thesis. The predominance of dissertations related to stricto sensu programs, within a specific subject, was also found in another bibliometric study, which found this to be true for $82 \%$ of dissertations found in the CAPES database on palliative care ${ }^{(43)}$. One possibility for this result is the fact that the master's courses were implemented first, and are in greater numbers among postgraduation programs, contributing annually to a vast scientific production ${ }^{(44)}$.

There was a diversity of study topics, including subjects such as the legalization of marijuana. However, most of these studies are focused on areas related to assistance to specific groups or conditions. This demonstrates consistency with the objective of an integrative review, which is to assist in decision making in clinical practice through the synthesis of available knowledge ${ }^{(1)}$.

The Universidade de São Paulo (USP) stood out in the number of works produced; its courses are between grades 6 and 7 in the CAPES assessment, and the institution is considered to be a leadership in the country due to its high standard. Similar results were found in a bibliometric study on the history of nursing, in a research in the CAPES database ${ }^{(45)}$. The fact that USP is a pioneer in offering postgraduation programs in the field of nursing, with a master's degree program since 1973 
and a PhD course since 1989, can explain its high production on varied themes ${ }^{(46)}$.

Although Mendes, Silveira and Galvão (2008) were the authors most used for methodological support, it was possible to notice that there are other national and international references. This is observed in a national dissertation, considered reference on the theme, which compared five studies on the subject and developed an instrument for data analysis, mentioning the contribution of stricto sensu production to the integrative review study design ${ }^{(47)}$.

The number of databases and sites used is quite large: 27. However, there is a predominance of the LILACS database, which was used in $86.1 \%$ of the studies. The countries (Latin America) considered by the database, the language, the inclusion of its acronym in the DeCS list and studies published exclusively on LILACS may justify its dissemination among Brazilian researchers ${ }^{(48)}$.

Some aspects of the methodology were not mentioned in the studies, making its replicability impossible and, thus, demonstrating weaknesses in the construction of the study. It is essential to cite and explain the data collection period, descriptors, exclusion criteria and publishing period. Methodological rigor is an important aspect of primary research and of review studies. All types of researches require a standard of excellence to guarantee their validity and scientific credibility so that the result brings significant contributions to clinical practice ${ }^{(2-3)}$.

There is a tendency to present the results in a table with a synthesis of the studies main information. This is a strategy that allows the reader to easily find study results and information. It is considered an important attribute, which facilitates communication using visual strategies to present information, enabling comparisons, identification of patterns, and divergences ${ }^{(3-4)}$. However, as expected in the analysis of an integrative review, the quantitative data is not analyzed in-depth, and a greater emphasis is put on qualitative data and discursive analyses. This can lead to questions about its quality, since the subjective component does not allow clarity about studies inferences ${ }^{(2)}$.

There were no restrictions to the number of texts included in the review. $5(14 \%)$ researches had a sample of less than 10 articles, and most had up to 20 (54\%). Likewise, in the literature, there is no clarity on the number of articles that should be included in an integrative review; the recommendation is to use all that are found, regardless of how many ${ }^{(1)}$.

The data collection method proposed and applied by Ursi (2005) was used in $44.4 \%$ of the studies, being the most cited reference. An instrument for data extraction prevents relevant information from being lost during the review, minimizes errors in transcription, guarantees reliability, and indicates how the data will be analyzed, facilitating its execution ${ }^{(2,4)}$.

Even with these findings, integrative review studies are still in low numbers in master's and doctorate scientific production in nursing, varying from less than $1 \%$ to $13 \%$ of the publications, depending on the theme ${ }^{(43,49)}$. However, an obstacle against conducting an integrative review in stricto sensu programs is the difficulty in publishing the paper, considering the current requirements related to scientific publications ${ }^{(2)}$.

Thus, it was concluded that the integrative review is a methodology that can assist in the progress of science and in the construction of knowledge in master's and doctoral programs in nursing. It can synthesize researches findings, point out gaps, justify new studies, and avoid repeating content $\mathrm{t}^{(1-2)}$.

\section{Study limitations}

The selection of a single database can be considered a limitation for this study, as other dissertations and theses are in other databases, and thus were not identified/selected. However, the study considered the main database for indexed graduate studies in Brazil.

\section{Contributions to the field of nursing, health, or public policy}

The study brings, as a contribution to the field of nursing and health, information for the development of integrative review studies, providing the researcher with elements to be able to respect the rigors of the method and its execution.

\section{CONCLUSIONS}

Despite the popularity of integrative review studies, they are undervalued in the stricto sensu postgraduation programs in Brazil. Shortcomings in the execution of the method, such as the absence of elements that constitute its basic steps, compromise the rigor of the studies and the compliance with the demands of scientific research. This is an important research method for professional training and clinical practice in nursing, and can be applied at different levels of education, contributing to the scientific diffusion in Brazilian nursing.

Analyzing the application of integrative reviews in dissertations and theses allowed to characterize their production, pointing out possibilities for expansion, mainly in doctorate courses, which can use this methodology to develop new knowledge, not only with philosophical foundations, but also through the intertwining of scientific knowledge and practice.

\section{REFERENCES}

1. Mendes KDS, Silveira RCCP, Galvão CM. Revisão integrativa: método de pesquisa para a incorporação de evidências na saúde e na enfermagem. Texto Contexto Enferm. 2008;17(4):758-64. doi: 10.1590/S0104-07072008000400018

2. Roman AR, Friedlander MR. Revisão integrativa de pesquisa aplicada à enfermagem. Cogitare Enfermagem [Internet]. 1998 [cited 2020 May 02];3(2):109-12. Available from: https://revistas.ufpr.br/cogitare/article/view/44358/26850.

3. Soares CB, Hoga LAK, Peduzzi M, Sangaleti C, Yonekura T, Silva DRA. Revisão Integrativa: Conceitos e métodos utilizados na enfermagem. Rev Esc Enferm USP [Internet]. 2014 [cited 2019 May 25];48(2):335-45. Available from: www.scielo.br/pdf/reeusp/v48n2/pt_0080-6234reeusp-48-02-335.pdf 
4. Souza MT, Silva, MD, Carvalho R. Revisão integrativa: o que é e como fazer. Einstein [Internet]. 2010 [cited 2019 May 25];8(1 Pt 1):102-6. Available from: www.scielo.br/pdf/eins/v8n1/pt_1679-4508-eins-8-1-0102

5. Gil AC. Como Elaborar Projetos de Pesquisa. 6a ed. São Paulo: Atlas; 2017. 192 p.

6. Winters JRF, Prado ML, Lazzari DD, Jardim VLT. Nursing higher education in MERCOSUR: a bibliometric study. Rev Bras Enferm. 2018;71(Suppl 4):1732-9. doi: 10.1590/0034-7167-2017-0405

7. Catoia EA. Caracterização da assistência à saúde prestada às pessoas privadas de liberdade que vivem com HIV/AIDS: uma revisão integrativa [Dissertação]. Ribeirão Preto: Universidade de São Paulo; 2014.

8. Pessoa FMI. Violência sofrida por mulheres antes do seu encarceramento: revisão integrativa da literatura [Dissertação]. Ribeirão Preto: Universidade de São Paulo; 2016.

9. Ignacio DS. Alta hospitalar responsável: em busca da continuidade do cuidado para pacientes em cuidados paliativos no domicílio, uma revisão integrativa [Dissertação]. Ribeirão Preto: Universidade de São Paulo; 2017.

10. Brito LSC. Evidências à intervenção de enfermagem "controle de infecção" da classificação das intervenções de enfermagem: orientação e ensino sobre a lavagem das mãos [Dissertação]. Botucatu: Universidade Estadual Paulista Júlio de Mesquita Filho; 2015.

11. Berlet LJ. Infecção no período puerperal: implicações para a enfermagem [Dissertação]. Rio de Janeiro: Universidade do Estado do Rio de Janeiro; 2015.

12. Rassele T. Recomendações dos cuidados de enfermagem ao paciente adulto submetido à terapia renal substitutiva na unidade de terapia intensiva [Dissertação]. Florianópolis: Universidade Federal de Santa Catarina; 2016.

13. Lopes VC. Conhecimento produzido por enfermeiros brasileiros sobre hipertensão arterial: estudo de revisão integrativa [Dissertação]. Guarulhos: Universidade Universus Veritas Guarulhos; 2014.

14. Jiticovski AFM. Hipertensão arterial em populações negras: análise dos artigos publicados no brasil e estados unidos por enfermeiros [Dissertação]. Guarulhos: Universidade Universus Veritas Guarulhos; 2014.

15. Alencar ACL. Tuberculose e diabetes mellitus: abordagens na trajetória espaço-temporal [Dissertação]. São Paulo: Universidade de São Paulo; 2014.

16. Lima MCRAD. Estratégias para o controle da tuberculose no sistema prisional: revisão integrativa da literatura [dissertação]. São Paulo: Universidade de São Paulo; 2015.

17. Pelaes CES. Métodos de ensino na prevenção de infecção da corrente sanguínea associada ao cateter: uma revisão integrativa da literatura [Dissertação]. São Paulo: Universidade de São Paulo; 2013.

18. Hara CYN. Tecnologia digital educacional e o estudo de caso clínico para a aprendizagem de estudantes de enfermagem: revisão integrativa [Dissertação]. Ribeirão Preto: Universidade de São Paulo; 2015.

19. Liberali M. Estratégias de ensino utilizadas no desenvolvimento do pensamento crítico na enfermagem: revisão integrativa [Dissertação]. Ribeirão Preto: Universidade de São Paulo; 2017.

20. Ilher AS. Práticas educativas para enfermeiros em serviço de emergência: uma revisão integrativa [dissertação]. São Leopoldo: Universidade do Vale do Rio dos Sinos; 2017.

21. Correa WCO. Gestão do cuidado por enfermeiras brasileiras: uma revisão integrativa da literatura [Dissertação]. Feira de Santana: Universidade Estadual de Feira de Santana; 2015.

22. Areco FS. A espiritualidade para mulheres com câncer de mama: uma revisão integrativa [Dissertação]. Ribeirão Preto: Universidade de São Paulo; 2016.

23. Abrahao CA. Estratégias de redução do estresse e seu impacto no sistema imune de mulher com câncer de mama: evidências para a prática clínica [Dissertação]. Ribeirão Preto: Universidade de São Paulo; 2013.

24. Bastos YS. Denver II: discrepâncias na avaliação do desenvolvimento da criança brasileira[Dissertação]. Guarulhos: Universidade Universus Veritas Guarulhos; 2017.

25. Peixoto RCBO. Estratégias do setor saúde no enfrentamento da violência doméstica infantil [Dissertação]. Guarulhos: Universidade Universus Veritas Guarulhos; 2017.

26. Quintana JM. Produção científica sobre quedas em idosos: componentes da classificação internacional de funcionalidade, incapacidade e saúde [Dissertação]. Rio Grande: Universidade Federal do Rio Grande; 2013.

27. Paula Junior NF. Estado da arte do evento quedas em idosos: uma revisão integrativa de literatura [Dissertação]. Florianópolis: Universidade Federal de Santa Catarina; 2014.

28. Cassola TP. Quedas em pacientes psiquiátricos: uma revisão integrativa [Dissertação]. Porto Alegre: Universidade Federal do Rio Grande do Sul; 2017.

29. Sartoreto IS. Satisfação e insatisfação no trabalho dos enfermeiros: revisão integrativa da literatura [Dissertação]. São Paulo: Universidade de São Paulo; 2013.

30. Leal JAL. Processo de trabalho da enfermeira em diferentes países [Tese]. Salvador: Universidade Federal da Bahia; 2016.

31. Abreu SP. Utilização de Instrumento de Classificação de Pacientes: Análise da Produção do Conhecimento Brasileira [Dissertação]. São José do Rio Preto: Faculdade de Medicina de São José do Rio Preto; 2014. 
32. Silva DDC. O raciocínio clínico e sua aplicação ao processo de diagnosticar em enfermagem - revisão integrativa de literatura [Dissertação]. Rio de Janeiro: Universidade Federal do Rio de Janeiro; 2016.

33. Weiber AFM. Legalização da maconha: uma revisão [Dissertação]. Jequié: Universidade Estadual do Sudoeste da Bahia; 2016.

34. Balthazar MAP. Cuidados com a saúde dos soldadores nos estaleiros brasileiros - uma revisão integrativa [Dissertação]. Niterói: Universidade Federal Fluminense; 2016.

35. Coelho ADA. Intervenções de enfermagem para tratamento de úlcera por pressão em pacientes acamados: revisão integrativa da literatura [Dissertação]. Fortaleza: Universidade Federal do Ceará; 2013.

36. Molina FMR. Cuidado de enfermagem ao receptor de transplante de fígado no período pós-operatório: revisão integrativa [Dissertação]. Ribeirão Preto: Universidade de São Paulo; 2014.

37. Magro JTJ. Sexualidade de candidatos e receptores de transplante de fígado: revisão integrativa da literatura [Dissertação]. Ribeirão Preto: Universidade de São Paulo; 2017.

38. Silva LG. Projeto terapêutico singular: uma revisão integrativa da literatura [Dissertação]. São Paulo: Universidade de São Paulo; 2015.

39. Gimenez LBH. O estresse precoce como um dos determinantes do uso abusivo de substâncias psicoativas: revisão integrativa [Dissertação]. Ribeirão Preto: Universidade de São Paulo; 2015.

40. Furquim PS. Tecnologia em saúde e segurança na administração de medicamentos em pacientes hospitalizados: uma revisão integrativa [Dissertação]. Ribeirão Preto: Universidade de São Paulo; 2015.

41. Hollanda TC. Magnet Recognition Program: revisão integrativa de literatura [Dissertação]. São Paulo: Universidade de São Paulo; 2015.

42. Silva ROC. Apoio matricial em saúde mental: uma possibilidade de educação permanente? [Dissertação]. Niterói: Universidade Federal Fluminense; 2017.

43. Almeida ARLP, Oliveira FA, Marinho CLA, Leite AMC, Silva RS. Enfermagem em cuidados paliativos nas dissertações e teses no Brasil: um estudo bibliométrico. Rev Min Enferm [Internet]. 2019 [cited 2020 May 02];23:e-1188. Available from: https://www.reme.org.br/exportarpdf/1331/e1188.pdf

44. Scochi CGS, Munari DB, Gelbcke FL, Erdmann AL, Gutiérrez MGR, Rodrigues RAP. Pós-graduação Stricto Sensu em enfermagem no Brasil: avanços e perspectivas. Rev Bras Enferm [Internet]. 2013 [cited 2020 May 02];66(esp):80-9. Available from: http://www.scielo.br/pdf/reben/ v66nspe/v66nspea11.pdf

45. Taffner VBM, Pimentel RRS, Valóta IAC, Ribeiro AAA, Silva LH da, Piber RS, Takashi MH, Freitas GF de. Teses e dissertações acerca da história da Enfermagem. Rev Enferm UFPE[Internet]. 2019 [cited 2020 May 02];13:e242905. Available from: https://periodicos.ufpe.br/revistas/ revistaenfermagem/article/download/242905/33631

46. Scochi CGS, Ferreira MA, Gelbcke FL. Relatório de Avaliação: Enfermagem. Ministério da Educação, Coordenação de Aperfeiçoamento de Pessoal de Nível Superior. Avaliação Quadrienal 2017 [Internet]. 2017 [cited 2019 May 25]. Available from: https://capes.gov.br/images/ stories/download/avaliacao/relatorios-finais-quadrienal-2017/20122017-ENFERMAGEM-quadrienal.pdf

47. Ursi ES. Prevenção de lesões de pele no perioperatório: revisão integrativa da literatura [Dissertação]. Ribeirão Preto: Universidade de São Paulo; 2005.

48. Nascimento LC, Moraes ER, Silva JC, Veloso LC, Vale ARMC. Cuidador de idosos: conhecimento disponível na base de dados LILACS. Rev Bras Enferm. 2008;61(4):514-17. doi: 10.1590/S0034-71672008000400019

49. Medeiros AB, Oliveira LV, Silva GWS, Lopes TRG, Carvalho JBL, Miranda FAN. Teses e dissertações da enfermagem brasileira sobre saúde da mulher no sistema prisional. Rev Rene [Internet]. 2019 [cited 2020 May 02];20:e41752. Available from: http://periodicos.ufc.br/rene/article/ view/41752/99453 